\title{
ON THE DEGREE SEQUENCE OF AN EVOLVING RANDOM GRAPH PROCESS AND ITS CRITICAL PHENOMENON
}

\author{
XIAN-YUAN WU, ${ }^{*}$ Capital Normal University \\ ZHAO DONG ${ }^{* * * * *}$ AND \\ KE LIU, ${ }^{* * * * * *}$ Chinese Academy of Sciences \\ KAI-YUAN CAI, ${ }^{* * * * *}$ Beijing University of Aeronautics and Astronautics
}

\begin{abstract}
In this paper we focus on the problem of the degree sequence for a random graph process with edge deletion. We prove that, while a specific parameter varies, the limit degree distribution of the model exhibits critical phenomenon.
\end{abstract}

Keywords: Degree sequence; power law; critical phenomenon; real-world networks

2000 Mathematics Subject Classification: Primary 05C07; 05C80

\section{Introduction and statement of the results}

In the past decade, a lot of effort has been devoted to studying large-scale real-world networks and modeling their properties. For a general introduction to this topic, we refer the reader to [1], [3], [7], [20], and [24]. Although the study of real-world networks as graphs can be traced back a long time, such as the classical model proposed by Erdös and Rényi [15] and Gilbert [16], recent influential activity perhaps started with the work of Watts and Strogatz [25] about the 'small-world phenomenon' published in 1998. Another influential work may be due to the scale-free model proposed by Bollobás and Albert [5] in 1999. Since then, various forms of scale-free phenomenon have been widely revealed. In particular, power-law degree distributions have been extensively investigated. Many new models have been introduced to circumvent the shortcomings of the classical models introduced by Erdös and Rényi [15] and Grilbert [16]. One class of these new models aimed to explain the underlying causes for the emergence of power-law degree distributions; see, for example, [8], [9], [11], [12], [13], [14], and [18].

\footnotetext{
Received 8 December 2008; revision received 24 July 2009.

* Postal address: School of Mathematical Sciences, Institute of Mathematics and Interdisciplinary Science, Capital Normal University, Beijing, 100048, China. Email address: wuxy@ mail.cnu.edu.cn

Supported in part by the Foundation of Beijing Education Bureau under grant 09224010003 and the Natural Science Foundation of China under grant 10971143.

** Postal address: Academy of Mathematics and System Sciences, Chinese Academy of Sciences, Beijing, 100190, China.

*** Email address: dzhao@amss.ac.cn

Supported in part by the Natural Science Foundation of China under grants 10671197 and 10721101.

**** Email address: kliu@ amss.ac.cn

Supported in part by the Natural Science Foundation of China under grants 60674082, 70221001, and 70731003.

***** Postal address: National Key Laboratory of Science and Technology on Integrated Control, Department of Automatic Control, Beijing University of Aeronautics and Astronautics, Beijing, 100083, China.

Email address: kycai@buaa.edu.cn

Supported in part by the Natural Science Foundation of China and Microsoft Research Asia under grant 60633010.
} 
Besides the power-law degree distributions (see [2] and [10]), other forms of the degree distributions can also be observed in real-world networks (see [4] and [22]). For example, Gaussian distributions can be observed in the acquaintance network of Mormons [6], and exponential distributions can be observed in the power grid of southern California [25]. On the other hand, the degree distribution of the network of world airports [4] interpolates between Gaussian and exponential distributions, whereas the degree distribution of the citation network in high energy physics [19] interpolates between exponential and power-law distributions. For more forms of degree distributions, we refer the reader to [21].

Different models often lead to different forms of degree distributions. An interesting problem arises naturally: does there exist some dynamically evolving random graph process which brings forth various degree distributions by continuous changing of its parameters only? To the best of the authors' knowledge, it seems that the problem and its answer have not been formulated in a mathematically rigorous manner. In this paper we focus on a model with edge deletions and provide precise analysis to show, while a parameter varies, that the model exhibits various degree distributions.

We begin by introducing our model and then state our main results. Consider the following process which generates a sequence of graphs $G_{t}=\left(V_{t}, E_{t}\right), t \geq 1$. Write $v_{t}=\left|V_{t}\right|$ and $e_{t}=\left|E_{t}\right|$.

Time step 1 . Let $G_{1}$ consist of an isolated vertex $x_{1}$.

Time step $t \geq 2$. (i) With probability $\alpha_{1}>0$ we add a vertex $x_{t}$ to $G_{t-1}$. We then add $m$ random edges incident with $x_{t}$. In the case in which $e_{t-1}>0$, the $m$ random neighbors $w_{1}, w_{2}, \ldots, w_{m}$ are chosen independently. For $1 \leq i \leq m$ and $w \in V_{t-1}$,

$$
\mathrm{P}\left(w_{i}=w\right)=\frac{d_{w}(t-1)}{2 e_{t-1}},
$$

where $d_{w}(t-1)$ denotes the degree of vertex $w$ at the beginning of substep $t$. Thus, neighbors are chosen by preferential attachment. In the case in which $e_{t-1}=0$, we add a new vertex $x_{t}$ and join it to a randomly chosen vertex in $V_{t-1}$.

(ii) With probability $\alpha-\alpha_{1} \geq 0$ we add $m$ random edges to existing vertices. If $e_{t-1}>0$ then both endpoints are chosen independently with the same probabilities as in (1.1). Otherwise, we do nothing.

(iii) With probability $1-\alpha \geq 0$ we delete $\min \left\{m, e_{t-1}\right\}$ randomly chosen edges from $E_{t-1}$.

Now we assume that

$$
\frac{1}{2}<\alpha \leq 1, \quad 0<\alpha_{1} \leq \alpha .
$$

For given $\alpha$ and $\alpha_{1}$ satisfying (1.2), define

$$
\alpha_{c}:=4 \alpha-2, \quad \eta:=\frac{m \alpha_{c}}{2},
$$

and choose $\epsilon=\epsilon\left(\alpha, \alpha_{1}\right) \in(0, \eta)$ such that

$$
\rho_{\epsilon}:=\max \left\{\frac{m\left(\alpha_{c}-\alpha_{1}\right)}{2(\eta-\epsilon)}, \frac{1}{2}\right\}<1 .
$$

Let

$$
\beta=\frac{\alpha_{c}}{\alpha_{c}-\alpha_{1}}, \quad \gamma=1-\frac{\alpha_{1}-\alpha_{c}}{2(1-\alpha)}, \quad \theta=\frac{2 \alpha_{c}-\alpha_{1}}{2 \alpha_{c}}, \quad \mu=\frac{\alpha_{c}}{2(1-\alpha)} .
$$


To obtain our main results, besides (1.2), we assume that

$$
\alpha_{1}<2 \alpha_{c}
$$

Now, let $D_{k}(t)$ be the number of vertices with degree $k \geq 0$ in $G_{t}$ and let $\bar{D}_{k}(t)$ be the expectation of $D_{k}(t)$.

The main results of this paper are as follows.

Theorem 1.1. Assume that (1.2) and (1.6) hold. Then $\alpha_{c}$ defined in (1.3) is a critical point for the degree sequence of the model satisfying the following conditions.

1. If $\alpha_{1}<\alpha_{c}$ then there exists a constant $C_{1}=C_{1}\left(m, \alpha, \alpha_{1}\right)$ such that, for any $v \in$ $\left(0,1-\rho_{\epsilon}\right)$,

$$
\left|\frac{\bar{D}_{k}(t)}{t}-C_{1} k^{-1-\beta}\right|=O\left(t^{\rho_{\epsilon}+v-1}\right)+O\left(k^{-2-\beta}\right) .
$$

2. If $\alpha_{1}>\alpha_{c}$ then there exists a constant $C_{2}=C_{2}\left(m, \alpha, \alpha_{1}\right)$ such that

$$
\left|\frac{\bar{D}_{k}(t)}{t}-C_{2} \gamma^{k} k^{-1+\beta}\right|=O\left(t^{-\theta}\right)+O\left(\gamma^{k} k^{-2+\beta}\right) .
$$

3. If $\alpha_{1}=\alpha_{c}$ then there exists a constant $C_{c}=C_{c}\left(m, \alpha, \alpha_{1}\right)$ such that, for any $v \in\left(0, \frac{1}{2}\right)$,

$$
\left|\frac{\bar{D}_{k}(t)}{t}-C_{c} u_{c}(k)\right|=O\left(t^{-1 / 2+v}\right)
$$

uniformly in $k$, where $u_{c}(k)=\int_{0}^{1} t^{k-1} \mathrm{e}^{-\mu /(1-t)} \mathrm{d} t$.

With help of computer calculation, we know that $u_{c}(k)$ satisfies

$$
\lim _{k \rightarrow \infty} \frac{\ln u_{c}(k)}{-k}=\lim _{k \rightarrow \infty} \frac{-\ln k}{\ln u_{c}(k)}=0 .
$$

Remark 1.1. Compared with the model $G\left(\alpha_{1}, \alpha-\alpha_{1}, 0,1-\alpha, m\right)$ in [12], Theorem 1.1 extends the range of power laws from the case $\alpha_{1}<2 \alpha-1$ to $\alpha_{1}<4 \alpha-2=\alpha_{c}$, where $\alpha_{c}$ is the critical point. Theorem 1.1 also extends the results of [14] with $\alpha_{0}=1-\alpha$; actually, Cooper [14] showed that, in case of $\alpha_{0}$ being small enough, the model possesses power-law degree distributions.

Based on Theorem 1.1, we can obtain the following two corollaries, which provide a complete distinction with respect to the parameters between the degree sequences for the present model.

Corollary 1.1. If the parameters satisfy

1. $\alpha>\frac{2}{3}$, or

2. $\alpha \leq \frac{2}{3}$ and $\alpha_{1}<\alpha_{c}$,

then the present random graph process has power-law degree sequence (1.7).

Corollary 1.2. Assume that $\alpha \leq \frac{2}{3}$.

1. If $\alpha_{c}<\alpha_{1}<2 \alpha_{c}$ then the present random graph process has exponential degree sequence (1.8).

2. If $\alpha_{1}=\alpha_{c}$ then the present random graph process has critical degree sequence (1.9). 
Remark 1.2. When $\alpha>\frac{2}{3}$, for any $\alpha_{1}$, the inequality $\alpha_{1} \leq \alpha<\alpha_{c}=4 \alpha-2$ holds always; therefore, part 1 of Corollary 1.1 follows from part 1 of Theorem 1.1. Parts 2 of Corollary 1.1 and Corollary 1.2 are straightforward from Theorem 1.1.

Remark 1.3. No result has been obtained for the case in which $\alpha \leq \frac{2}{3}$ and $2 \alpha_{c} \leq \alpha_{1} \leq \alpha$. Clearly, this case can only appear when $\alpha \leq \frac{4}{7}$. It is natural to conjecture that the model possesses an exponential degree sequence in this case.

\section{Proof of Theorem 1.1}

We first note that the methodology of our proof follows the standard procedure, which can be found in [13] and [14].

For times $s$ and $t$ with $1 \leq s \leq t$, let $d_{x_{s}}(t)$ be the degree of vertex $x_{s}$ in $G_{t}$. If $x_{s}$ is not added in time step $s$, i.e. at time step $s$ one of the other two substeps is executed, set $d_{x_{s}}(t)=0$.

Cooper et al. [14] derived

$$
\left|v_{t}-\alpha_{1} t\right| \leq c t^{1 / 2} \log t \quad \text { quite surely }
$$

for any constant $c>0$. We say that an event happens quite surely (q.s.) if the probability of the complimentary set of the event is $O\left(t^{-K}\right)$ for any $K>0$. The estimate for $e_{t}$ can be derived by the similar argument that

$$
\left|e_{t}-\eta t\right| \leq c t^{1 / 2} \log t \quad \text { q.s. }
$$

for any constant $c>0$.

By a standard argument in large deviation theory (see [23]), we can further show that, for any $\epsilon>0$, there exist $c_{1}, c_{2}>0$ such that

$$
\mathrm{P}\left(\left|e_{t}-\eta t\right| \geq \epsilon t\right) \leq c_{1} \exp \left\{-c_{2} t\right\}
$$

for all $t \geq 1$.

The following is our bounding for $d_{x_{s}}(t)$; note that our result is based on the exact estimation (2.2) for $e_{t}$.

Lemma 2.1. For any $\alpha \in\left(\frac{1}{2}, 1\right]$ and $\alpha_{1} \in(0, \alpha]$,

$$
d_{x_{s}}(t) \leq\left(\frac{t}{s}\right)^{\rho_{\epsilon}}(\log t)^{3} \quad \text { q.s. }
$$

where $\rho_{\epsilon}$ is given in (1.4).

Proof. Fix $s \leq t$. Suppose that $x_{s}$ is added in time step $s$. Let $X_{\tau}=d_{x_{s}}(\tau)$ for $\tau=s$, $s+1, \ldots, t$, and let $Y$ be the $\{1,2,3\}$-valued random variable with

$$
\mathrm{P}(Y=1)=\alpha_{1}, \quad \mathrm{P}(Y=2)=\alpha-\alpha_{1}, \quad \mathrm{P}(Y=3)=1-\alpha .
$$

Conditional on $X_{\tau}=x$ and $e_{\tau} \geq m$, we have

$$
X_{\tau+1}=x+\mathbf{1}_{\{Y=1\}} B\left(m, \frac{x}{2 e_{\tau}}\right)+\mathbf{1}_{\{Y=2\}} B\left(2 m, \frac{x}{2 e_{\tau}}\right)-\mathbf{1}_{\{Y=3\}} S\left(m, \frac{x}{e_{\tau}}\right),
$$

where $B(m, p)$ is the binomial random variable with parameter $(m, p)$ and $S\left(m, x / e_{\tau}\right)$ is the super geometric random variable with parameter $\left(e_{\tau}, x, m\right)$. 
If $e_{\tau}$ in (2.4) is substituted by $\eta \tau,(2.3)$ can be derived by a standard argument which is given in the proof of Lemma 3.1 of [14]. Actually, with the exact estimate (2.2) of $e_{t}$, (2.3) follows from a random modification of such a standard argument. Details can be found in [26].

Now we follow the basic procedures in [14] to establish the recurrence for $\bar{D}_{k}(t)$. Set $D_{-1}(t)=0$ for all $t \geq 1$. For $k \geq 0$, we have

$$
\begin{aligned}
\bar{D}_{k}(t+1)= & \bar{D}_{k}(t) \\
& +\left(2 \alpha-\alpha_{1}\right) m \mathrm{E}\left(-\frac{k D_{k}(t)}{2 e_{t}}+\frac{(k-1) D_{k-1}(t)}{2 e_{t}}+O\left(\frac{\Delta_{t}}{e_{t}}\right) \mid e_{t}>0\right) \mathrm{P}\left(e_{t}>0\right) \\
& +(1-\alpha) m \mathrm{E}\left(\frac{(k+1) D_{k+1}(t)}{e_{t}}-\frac{k D_{k}(t)}{e_{t}}+O\left(\frac{\Delta_{t}}{e_{t}}\right) \mid e_{t} \geq m\right) \mathrm{P}\left(e_{t} \geq m\right) \\
& +\alpha_{1} \mathbf{1}_{\{k=m\}} \mathrm{P}\left(e_{t}>0\right)+O\left(\mathrm{P}\left(e_{t}=0\right)\right)+O\left(\mathrm{P}\left(e_{t}<m\right)\right)
\end{aligned}
$$

where $\Delta_{t}$ denotes the maximum degree in $G_{t}$ and the term $O\left(\Delta_{t} / e_{t}\right)$ accounts for the probability that we create larger than one degree changes for some vertices at time step $t+1$. By (2.2) and Lemma 2.1, we have

$$
\frac{\Delta_{t}}{e_{t}} \leq O\left(t^{\rho_{\epsilon}-1}(\log t)^{3}\right) \quad \text { q.s. }
$$

With the help of (2.1), (2.2), (2.5), and Lemma 2.1, we obtain the recurrence for $\bar{D}_{k}(t)$ as follows: $\bar{D}_{-1}(t)=0$ for all $t>0$ and, for $k \geq 0$,

$$
\begin{aligned}
\bar{D}_{k}(t+1)= & \bar{D}_{k}(t)+\left(A_{2}(k+1)+B_{2}\right) \frac{\bar{D}_{k+1}(t)}{t}+\left(A_{1} k+B_{1}+1\right) \frac{\bar{D}_{k}(t)}{t} \\
& +\left(A_{0}(k-1)+B_{0}\right) \frac{\bar{D}_{k-1}(t)}{t}+\alpha_{1} \mathbf{1}_{\{k=m\}}+O\left(t^{\rho_{\epsilon}-1}(\log t)^{3}\right),
\end{aligned}
$$

where

$$
\begin{gathered}
A_{2}=\frac{1-\alpha}{2 \alpha-1}, \quad A_{1}=-\frac{2-\alpha_{1}}{2(2 \alpha-1)}, \quad A_{0}=\frac{2 \alpha-\alpha_{1}}{2(2 \alpha-1)}, \\
B_{2}=B_{0}=0, \quad \text { and } \quad B_{1}=-1 .
\end{gathered}
$$

Note that the term $O\left(t^{\rho_{\epsilon}-1}(\log t)^{3}\right)$ in (2.6) is independent of $k$, which follows from the fact that $e_{t}=O(t)$ and $k D_{k}(t) \leq 2 e_{t}=O(t)$ uniformly in $k$.

Recurrence (2.6) corresponds to the following recurrence in $k: d_{-1}=0$ and, for $k \geq-1$,

$$
\left(A_{2}(k+2)+B_{2}\right) d_{k+2}+\left(A_{1}(k+1)+B_{1}\right) d_{k+1}+\left(A_{0} k+B_{0}\right) d_{k}=-\alpha_{1} \mathbf{1}_{\{k=m-1\}} .
$$

The following lemma shows that (2.7) is a good approximation to (2.6).

Lemma 2.2. Let $d_{k}$ be a solution to (2.7) such that $\left|d_{k}\right| \leq C / k$ for $k>0$ and a constant $C$. Then

1. if $\alpha_{1} \leq \alpha_{c}$, for any $v \in\left(0,1-\rho_{\epsilon}\right)$, there exists a constant $M_{1}>0$ such that

$$
\left|\bar{D}_{k}(t)-t d_{k}\right| \leq M_{1} t^{\rho_{\epsilon}+v}
$$

for all $t \geq 1$ and $k \geq-1$, 
2. if $\alpha_{c}<\alpha_{1}<2 \alpha_{c}$, there exists a constant $M_{2}>0$ such that

$$
\left|\bar{D}_{k}(t)-t d_{k}\right| \leq M_{2} t^{1-\theta}
$$

for all $t \geq 1$ and $k \geq-1$, where $\theta$ is given in (1.5).

Proof. This proof follows the methodology of Cooper et al. [14]. Specifically, we provide some details in order to analyze the case in which

$$
0<\varepsilon_{0}:=A_{2}+B_{1}+1-A_{0}=\frac{\alpha_{1}-\alpha_{c}}{\alpha_{c}}<1,
$$

which provides very important evidence for revealing the critical phenomenon. Note that Cooper et al. [14] only dealt with the case in which $\varepsilon_{0} \leq 0$.

Let $\Theta_{k}(t)=\bar{D}_{k}(t)-t d_{k}$, and let $k_{0}=k_{0}(t)=\left\lfloor t^{\rho_{\epsilon}}(\log t)^{3}\right\rfloor$. Lemma 2.1 implies that

$$
0 \leq \bar{D}_{k}(t) \leq t^{-10} \text { for } k \geq k_{0}(t)
$$

1. Equation (2.10) and $d_{k} \leq C / k$ imply that (2.8) holds for $k \geq k_{0}$ uniformly, i.e. there exists a constant $N_{1}>0$, independent of $k$ and $t$, such that

$$
\left|\bar{D}_{k}(t)-t d_{k}\right|=\left|\Theta_{k}(t)\right| \leq N_{1} t^{\rho_{\epsilon}}
$$

for all $k \geq k_{0}(t)$ and $t \geq 1$.

Let $L$ be the hidden constant in $O\left(t^{\rho_{\epsilon}-1}(\log t)^{3}\right)$ of (2.6). For any $v \in\left(0,1-\rho_{\epsilon}\right)$, let $R \geq L$, satisfying

$$
L t^{\rho_{\epsilon}-1}(\log t)^{3} \leq R t^{\rho_{\epsilon}+v-1}
$$

for all $t \geq 1$. Let $N_{2}=R /\left(\rho_{\epsilon}+v\right)+1$, take $\sigma>0$ such that

$$
1-\frac{R}{N_{2}}-(1+\sigma)\left(1-\rho_{\epsilon}-v\right) \geq 0
$$

and take $\delta \in(0,1)$ such that

$$
\delta^{1+\sigma}<\mathrm{e}^{-1}<\delta
$$

Let $t_{1}>0$ be an integer such that

$$
k_{0}(t) \leq-\frac{t}{A_{1}}=\frac{2(2 \alpha-1)}{2-\alpha_{1}} t
$$

and

$$
\delta^{1+\sigma} \leq\left(1-\frac{1}{t+1}\right)^{t+1}, \quad\left(1-\frac{1-R / l}{t+1}\right)^{(t+1) /(1-R / l)} \leq \delta,
$$

for all $t \geq t_{1}$ and $l \geq N_{2}$.

Now, for the above $t_{1}$, let $N_{3} \geq N_{1}$, satisfying

$$
\left|\Theta_{k}(t)\right| \leq N_{3} t^{\rho_{\epsilon}+v} \quad \text { for all } 1 \leq t \leq t_{1} \text { and } k \geq-1 .
$$

Take

$$
M_{1}=\max \left\{N_{2}, N_{3}\right\}
$$


We will prove that (2.8) holds for the above $M_{1}$ by induction. Our inductive hypothesis is

$$
\mathscr{H}_{t}^{1}:\left|\Theta_{k}(t)\right| \leq M_{1} t^{\rho_{\epsilon}+v} \quad \text { for all } k \geq-1 .
$$

Note that (2.14) and (2.15) imply that $\mathscr{H}_{t}^{1}$ holds for $1 \leq t \leq t_{1}$.

It follows from (2.6) and (2.7) that

$$
\begin{aligned}
\Theta_{k}(t+1)= & \Theta_{k}(t)+A_{2}(k+1) \frac{\Theta_{k+1}(t)}{t}+\left(A_{1} k+B_{1}+1\right) \frac{\Theta_{k}(t)}{t} \\
& +A_{0}(k-1) \frac{\Theta_{k-1}(t)}{t}+O\left(t^{\rho_{\epsilon}-1}(\log t)^{3}\right) .
\end{aligned}
$$

For $t \geq t_{1}$, by (2.12), we have $t+A_{1} k+B_{1}+1 \geq 0$ and then (2.16) implies that

$$
\left|\Theta_{k}(t+1)\right| \leq\left(t+\varepsilon_{0}\right) M_{1} t^{\rho_{\epsilon}+v-1}+R t^{\rho_{\epsilon}+v-1} .
$$

Since $\alpha_{1} \leq \alpha_{c}$, we have $\varepsilon_{0} \leq 0$. Then, combining (2.11), (2.13), and (2.15), we have

$$
\frac{\left(t+\varepsilon_{0}\right) M_{1} t^{\rho_{\epsilon}+v-1}+R t^{\rho_{\epsilon}+v-1}}{M_{1}(t+1)^{\rho_{\epsilon}+v}} \leq \delta^{\left(1-R / M_{1}-(1+\sigma)\left(1-\rho_{\epsilon}-v\right)\right) /(t+1)} \leq 1 .
$$

The induction hypothesis $\mathscr{H}_{t+1}^{1}$ has been verified and the proof of part 1 is complete.

2. In this case we have $\alpha_{c}<\alpha_{1}<2 \alpha_{c}$, and then, for some $v \in\left(0, \frac{1}{2}\right), \varepsilon_{0} \leq \rho_{\epsilon}+v<1-\theta$ (note that in this case $\rho_{\epsilon}=\frac{1}{2}$ ). Proceeding as in the proof of part 1, for certain $\sigma>0$ and $\delta \in\left(\mathrm{e}^{-1}, 1\right)$, we have

$$
\frac{\left(t+\varepsilon_{0}\right) M_{2} t^{-\theta}+R t^{-\theta}}{M_{2}(t+1)^{1-\theta}} \leq \delta^{\left(1-\varepsilon_{0}-R / M_{2}-(1+\sigma) \theta\right) /(t+1)} \leq 1
$$

for sufficient large $t$ and $M_{2}$. This is enough for an inductive proof of (2.9).

Remark 2.1. Lemma 2.2 remains open for the case in which $\varepsilon_{0} \geq 1$, and this leads to condition (1.6).

Proof of Theorem 1.1. Theorem 1.1 is proved in two steps. Firstly, the Laplace method (see [17]) is used to solve (2.7) in the following three cases: (i) $\alpha_{1}<\alpha_{c}$, (ii) $\alpha_{1}>\alpha_{c}$, and (iii) $\alpha_{1}=\alpha_{c}$. Secondly, the resulting solutions of (2.7) are checked to see if they satisfy the requirements of Lemma 2.2. For details, we refer the reader to [26].

\section{Acknowledgements}

This work began with a visit by the first author to Institute of Mathematics, Academia Sinica. He is thankful to the probability group of IM-AS for their hospitality. The authors thank Colin Cooper for answering their questions on establishing recurrence (2.6).

\section{References}

[1] Albert, R. and Barabási, A.-L. (2002). Statistical mechanics of complex networks. Rev. Modern Phys. 74, 47-97.

[2] Albert, R., Barabási, A. and Jeong, H. (1999). Diameter of the World Wide Web. Nature 401, 130-131.

[3] Aiello, W., Chung, F. And Lu, L. (2002). Random evolution in massive graphs. In Handbook of Massive Data Sets, eds J. Abello et al., Kluwer, Dordrecht, pp. 510-519.

[4] Amaral, L. A. N., Scala, A., Barthélémy, M. and Stanley, H. E. (2000). Classes of small-world networks. Proc. Nat. Acad. Sci. USA 97, 11149-11152. 
[5] Barabási, A.-L. ANd Albert, R. (1999). Emergence of scaling in random networks. Science 286, 509-512.

[6] Bernard, H. R. et al. (1988). Studying social relations cross-culturally. Ethnology 27, 155-179.

[7] Bollobás, B. ANd Riordan, O. (2002). Mathematical results on scale-free random graphs. In Handbook of Graphs and Networks, John Wiley, Berlin, pp. 1-34.

[8] Bollobás, B. and Riondan, O. (2004). The diameter of a scale-free random graph. Combinatorica 4, 5-34.

[9] Bollobás, B., Riordan, O., Spencer, J. And Tusnády, G. (2001). The degree sequence of a scale-free random graph process. Random Structures Algorithms 18, 279-290.

[10] Broder, A. et al. (2000). Graph structure in the Web. In Proc. 9th Internat. World Wide Web Conf. Comput. Networks, North-Holland, Amsterdam, pp. 309-320.

[11] Buckley, P. G. AND Osthus, D. (2004). Popularity based random graph models leading to a scale-free degree sequence. Discrete Math. 282, 53-68.

[12] Chung, F. And Lu, L. (2004). Coupling online and offline analysis for random power law graphs. Internet Math. 1, 409-461.

[13] Cooper, C. ANd Frieze, A. (2003). A general model of undirected web graphs. Random Structures Algorithms 22, 311-335.

[14] Cooper, C., Frieze, A. AND Vera, J. (2004). Random deletion in a scale-free random graph process. Internet Math. 1, 463-483.

[15] Erdös, P. And RÉnyi, A. (1959). On random graphs. I. Publ. Math. Debrecen 6, 290-297.

[16] Gilbert, E. N. (1959). Random graphs. Ann. Math. Statist. 30, 1141-1144.

[17] Jordan, C. (1939). Calculus of Finite Differences. Hungarian Agent Eggenberger Book-Shop, Budapest.

[18] Kumar, R. et al. (2000). Stochastic models for the web graph. In 41st Annual Symp. Foundations Comput. Sci. (Redondo Beach, CA, 2000), IEEE Computer Society, Los Alamitos, CA, pp. 57-65.

[19] Lehmann, S., Lautrup, B. And Jackson, A. D. (2003). Citation networks in high energy physics. Phys. Rev. E 68, 026113.

[20] Newman, M. E. J. (2003). The structure and function of the complex networks. SIAM Rev. 45, 167-256.

[21] Scala, A., Amaral, L. A. N. and Barthélémy, M. (2001). Small-world networks and the conformation space of a short lattice polymer chain. Europhys. Lett. 55, 594-600.

[22] Strogatz, S. H. (2001). Exploring complex networks. Nature 410, 268-276.

[23] Stroock, D. W. (1984). An Introduction to the Theory of Large Deviations. Springer, New York.

[24] Watts, D. J. (1999). Small Worlds. Princeton University Press.

[25] Watts, D. J. and Strogatz, S. H. (1998). Collective dynamics of 'small-world' networks. Nature 393, 440-442.

[26] Wu, X.-Y., Dong, Z., LiU, K. And CAI, K.-Y. (2008). On the degree sequence and its critical phenomenon of an evolving random graph process. Preprint. Available at http://arxiv.org/abs/0806.4684v1. 